\title{
All polarization-maintaining passively mode- locked ytterbium-doped fiber lasers, behavior under two different cavity configurations
}

\author{
C. Cuadrado-Laborde ${ }^{1,2,3,{ }^{*}}$, A. Carrascosa ${ }^{1}$, A. Díez $^{1}$, J.L. Cruz $^{1}$, and M.V. Andrés ${ }^{1}$ \\ ${ }^{1}$ Departamento de Física Aplicada, ICMUV, Universidad de Valencia, Dr. Moliner 50, Burjassot E-46100, \\ Spain \\ ${ }^{2}$ Instituto de Física Rosario (CONICET-UNR), Blvr. 27 de Febrero 210bis, S2000EZP, Rosario, \\ Argentina, \\ ${ }^{3}$ Pontificia Universidad Católica Argentina, Facultad de Química e Ingeniería, Av. Pellegrini 3314, 2000, \\ Rosario, Argentina
}

*Corresponding author: christian.cuadrado@uv.es

\begin{abstract}
In this work, we review our recent investigations on the behavior of a polarizationmaintaining passively mode-locked ytterbium-doped laser in two different cavity configurations, namely: fiber-ring (FR) and Fabry-Perot (FP). Opposed to standard configurations that rely on the use of strong filtering within the cavity by including an ad hoc component with this purpose, here the filtering action is solely performed by the spectral overlapping of the different components within the fiber lasers. We found that the lack of a specific filter within the cavity does not deteriorate the performance as compared with previous works. We also report the changes in the output light pulses when the net dispersion of the cavity was varied. Additionally, different lengths of an ad hoc anomalous polarization-maintaining (PM) photonic crystal fiber (PCF) were used as intracavity dispersion compensator, to shift the operation of the laser from net-normal to the netanomalous regime. The shortest output light pulses [6 ps (FR) and 8 ps (FP)] were obtained when the net-cavity dispersion approached zero. Since the obtained light pulses were far to be transform-limited, we also discuss the possibility of out-of-cavity recompression by using the same PM-PCF mentioned above. After recompression, pulse widths of 3 ps were obtained, limited by the available length of PM PCF.
\end{abstract}

Index Terms: fiber laser, ytterbium, mode-locking, polarization maintaining, photonic crystal fibers.

\section{Introduction}

Short light pulses are now routinely obtained by soliton mode-locking [1, 2]. At the erbium emission wavelength, the necessary precise balance between chromatic dispersion and nonlinearity is easily achieved, since silica fibers present at this wavelength an anomalous chromatic dispersion. However, at the ytterbium emission wavelength, conventional single-mode fibers and fiber components present normal dispersion. Therefore, standard soliton mode-locking is only possible by including within the cavity a dispersioncompensation, which shifts the chromatic dispersion from normal to the net-anomalous regime. Unfortunately, at the expense of higher power loss or even losing the all-fiber configuration.

On the other hand, dissipative optical solitons (DS) in a nonlinear medium are confined wave packets of light whose existence and stability depend essentially on the energy balance [3]. In this work, we will refer specifically to DSs generated in all-normal-dispersion (ANDi) cavities. In contrast to solitons, which are Fourier-transform-limited; DSs are positively 
chirped pulses, which can be recompressed outside the cavity into the ultrashort regime [4, 5]. When compared with standard solitons, DSs are solutions of the cubic complex GinzburgLandau equation offering higher energies and stability, avoiding the challenge to provide lowloss net-anomalous dispersion at the ytterbium emission wavelength.

Industry adoption of mode locked fiber lasers is restrained by their sensitivity against external perturbations. Indeed, thermal and mechanical stress, ever-present in said environments, is known to influence the birefringence properties of fiber cavities, potentially resulting in unpredictable degradation of device performance unless the cavity is constructed exclusively out of polarization-maintaining (PM) fiber components. As a research group focused on the applications of fiber optics with more than twenty years of expertise, we are especially concerned about this problem. Therefore, our designs in this research area generally fulfill two synergic characteristics: on the one hand they are all-fiber and on the other, polarization-maintaining. Over the years, we have designed and worked with all kinds of fiber lasers, and when the fiber cavity is entirely assembled of PM fibers and components, it results in a notably improved behavior [6]. These PM fiber lasers are truly turn-on and forgetabout-it units. Further, there are several works in the literature experimentally proving the robustness of all-PM fiber laser cavities under thermal changes, vibrations and long-term stability. Merely as an example, in Ref. [7] the environmental robustness of a PM fiber laser was proven by placing the whole fiber cavity on a vibrating table and measuring the average output power, the mode-locking state was preserved over $40 \mathrm{~h}$ (lapse of time of the experiment). In the same work, the robustness against thermal variations was also studied by placing the whole fiber laser on a heating stage; only a slight and linear increment on the average output was measured in a temperature range from $22^{\circ} \mathrm{C}$ to $58^{\circ} \mathrm{C}$. Finally, the longterm stability was measured up to $80 \mathrm{~h}$ without noticeable degradation in the mode-locking regime. In short, a polarization-preserving design is always the best choice, whenever the objective is to develop environmentally robust fiber lasers, insensitive against temperature variations and mechanical vibrations.

Here we review our most recent works in the field of passively mode-locked ytterbiumdoped fiber lasers delivering DSs light pulses, in two different cavity configurations, namely fiber-ring and Fabry-Perot [8, 9]. Further, the different DSs PM fiber lasers presented to date all have in common the use of strong filtering within the cavity by using either bandpass pigtailed PM filters, tilted fiber Bragg gratings, or chirped fiber Bragg gratings [10, 11, 12, 13, $14,15,16,17]$. Examples in the literature without a specific filter in an ANDi PM Yb-doped fiber laser are scarce, and with output light pulses well in the nanoseconds range [18]. On the contrary, in our approach, we precluded this element, to simplify further the cavity. Therefore, here the filtering action is solely performed by the spectral overlapping of the different components within the fiber laser cavity. We also report the changes in the output light pulses when the net dispersion of the cavity was varied. Additionally, we shifted the operation of the laser from the net-normal to the net-anomalous regime; by using different lengths of an ad hoc anomalous PM photonic crystal fiber (PCF) as intracavity dispersion compensator. We used the same PM PCF for out-of-cavity recompression experiments.

\section{Experimental Details}

In Fig. 1 we show the schematic diagrams of the mode-locked lasers when the configuration of the cavity is a fiber-ring and a Fabry-Perot; see Figs. 1(a) and 1(b), respectively. We start by describing first the fiber-ring cavity of Fig. 1(a). The gain was provided by $0.47 \mathrm{~m}$ long PM ytterbium highly-doped, single-clad, optical fiber (PM YDF) (YDFSM-6/125 panda clad shape by NOVAE, core absorption > $700 \mathrm{~dB} / \mathrm{m}$ at $975 \mathrm{~nm}$, and numerical aperture of $0.16 \pm 0.02$ ). The PM YDF was pumped through a polarizationmaintaining wavelength division multiplexer (PM WDM, $980 \mathrm{~nm} / 1060 \mathrm{~nm}$ ) by a $976 \mathrm{~nm}$ emission wavelength pigtailed laser diode, providing a maximum pump power of $500 \mathrm{~mW}$. Next, at the output of the PM YDF was sandwiched a saturable absorber in transmission (SA) (BATOP $\mathrm{GmbH}$, high reflection band $1050 \mathrm{~nm} \leq \lambda \leq 1090 \mathrm{~nm}$, relaxation time constant $500 \mathrm{fs}$, absorptance $35 \%$, modulation depth $13 \%$, and saturation fluence of $300 \mu \mathrm{J} / \mathrm{cm}^{2}$ ) between 
two standard FC-PC fiber connectors, in order to guarantee the all-fiber structure of the laser. At the output of the SA was fusion-spliced the input of a polarization-maintaining optical isolator (PM ISO) (center wavelength $1064 \mathrm{~nm}$, fast axis blocked, > $22 \mathrm{~dB}$ extinction ratio). In turn, the output of the PM ISO was fusion-spliced to the input of an 80/20 polarizationmaintaining optical fiber coupler (PM OFC). The fiber-ring cavity was closed by using a delay line, which was fusion spliced between the $80 \%$ output of the PM OFC and the remaining port of the PM WDM. The output of this laser was obtained through the $20 \%$ port of the PM OFC.

On the other hand, in the Fabry-Perot configuration which is shown in Fig. 1(b), the fiber components were exactly the same; i.e., the same pump laser, PM YDF, PM WDM, and PM OFC. Therefore, it is not necessary to repeat its technical characteristics, and we will only describe the assembly of the cavity and the technical characteristics of those components which were not necessary above. The PM YDF was pumped through a PM WDM. Next, the PM YDF was spliced to the input port of the $80 / 20$ PM OFC. The $80 \%$ port of the PM OFC was in turn spliced to the semiconductor saturable absorber mirror (SESAM) (Batop GmbH, high reflection band $1010 \mathrm{~nm} \leq \lambda \leq 1110 \mathrm{~nm}$, relaxation time constant $1 \mathrm{ps}$ ). The cavity was closed by connecting the remaining port of the PM WDM to a PM fiber-pigtailed dichroic mirror (80\% reflectivity at $1030 \mathrm{~nm}$ ). Additionally, it was also included a delay line, between the PM YDF and the input of the PM OFC. Finally, the output of this laser is obtained through the 20 $\%$ port of the PM OFC; where a fiber pigtailed PM ISO was also included in order to avoid unwanted reflections into the cavity.

In some experiments, which will be specified whenever necessary, we insert in the cavity a PM delay line; see Figs. 1(a) and 1(b). This PM delay could be a certain length of a PM SMF (PM980-XP by Nufern, a cut-off wavelength of $920 \pm 50 \mathrm{~nm}$, and numerical aperture of 0.120 ) or an ad hoc polarization-maintaining photonic crystal fiber (PM PCF) specially designed and fabricated in our facilities $[19,20]$. Figure 1(c) shows as an inset a photograph of the cross-section of the PM PCF by using a scanning electron microscopy; the strong geometrical anisotropy observed is ultimately responsible for the polarization maintenance. The presence of the delay line allows changing the net dispersion of the cavity from different values of the net-normal to the net-anomalous regimes. Figure 1(c) also shows the experimental setup for out-of-cavity pulse recompression; the presence of the polarizationmaintaining optical circulator (PM OC) and the dichroic mirror ( $80 \%$ transmissivity), allows us to simultaneously testing single and double passes through the PM PCF.

It is also necessary to remark the absence of a filter within the cavity in both cavities; see Figs. 1(a) and 1(b). The filtering performance of the different components within the cavity should not be seriously taken into account due to its broadband characteristics, being finally the spectral overlapping between them the final responsible for both the emission wavelength and spectral bandwidth. In both setups, the output light pulses were monitored by using a 60 $\mathrm{GHz}$ sampling oscilloscope provided with a fast built-in photodetector $(53 \mathrm{GHz})$, real-time 2.5 $\mathrm{GHz}$ bandwidth oscilloscope, intensity autocorrelator (maximum scan range $200 \mathrm{ps}$ ), and an optical spectrum analyzer (wavelength accuracy $>20 \mathrm{pm}$ ). 

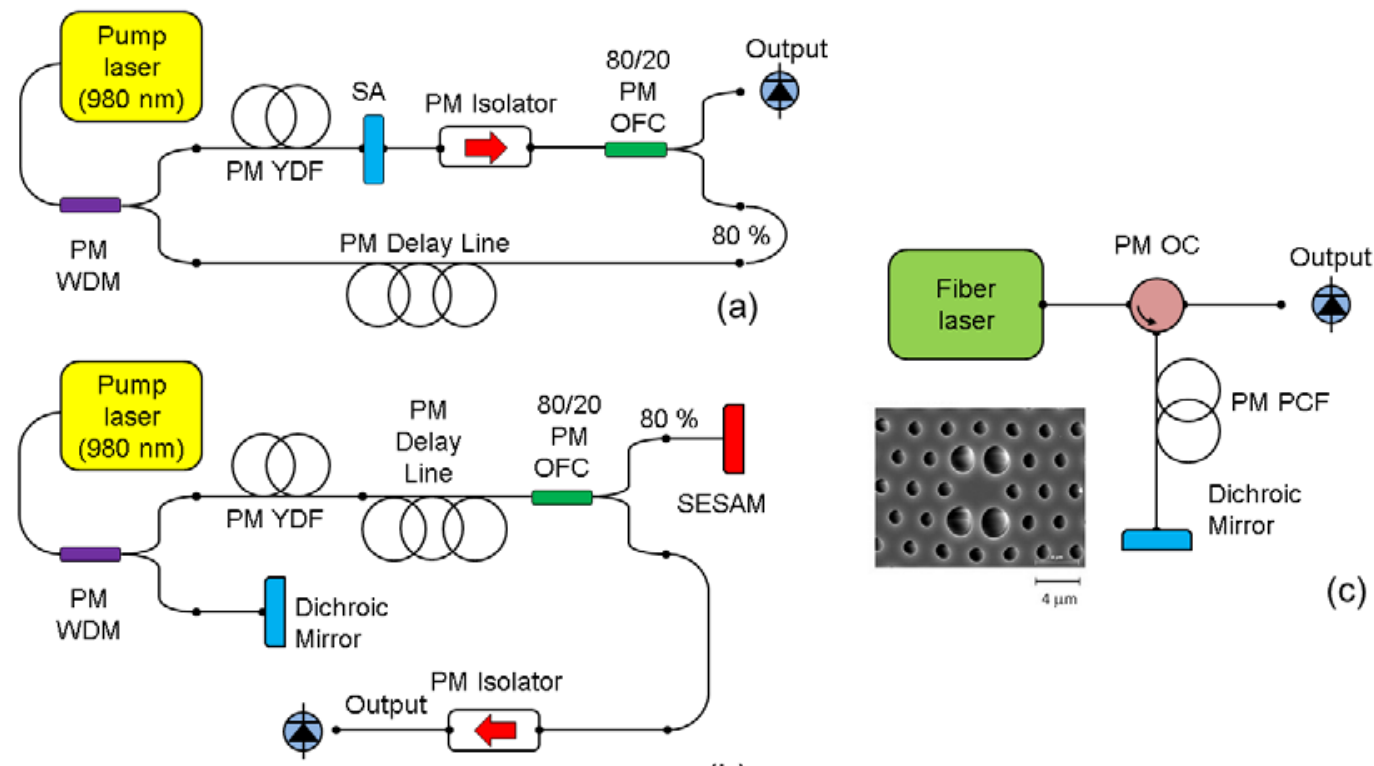

(b)

Fig. 1. Experimental laser setup when the selected cavity is: fiber-ring (a) and Fabry-Perot (b), where SA, PM OFC, and PM WDM stands for saturable absorber, polarization-maintaining optical fiber coupler, and polarizationmaintaining wavelength division multiplexer, respectively. (c) Experimental setup for out-of-cavity recompression; the photograph is a scanning electron microscope image of a transversal slide of the PM PCF. The PM OC allows single and double-pass through the PM PCF.

The chromatic dispersions of both the PM SMF and the PM PCF were measured in our laboratory by the interferometric technique [21] resulting in $24 \mathrm{ps}^{2} / \mathrm{km}$, and $-9 \mathrm{ps}^{2} / \mathrm{km}$, respectively, at $1030 \mathrm{~nm}$; whereas we estimated the dispersion of the PM YDF was of 25 $\mathrm{ps}^{2} / \mathrm{km}$. On the other hand, the power losses during each round trip of the light pulses were not significant, which is one of the main advantages of a full in-fiber configuration. Specifically, the total combined discrete-distributed power losses within one round-trip were below $4 \mathrm{~dB}$ and $6.7 \mathrm{~dB}$, when either PM SMF or PM PCF were used as delay lines for the fiber-ring cavity, respectively; whereas in the Fabry-Perot cavity the power losses were below $7.4 \mathrm{~dB}$.

As it was mentioned above, in both configurations we performed some modifications within the cavity in order to observe the changes in the output light pulses when the netdispersion or the medium gain was varied. For this reason, we start in both cases by describing the original configuration. In the case of the fiber-ring cavity, see Fig. 1(a); the original configuration consisted of a PM YDF length of $0.47 \mathrm{~m}$, and an extra PM SMF delay line length of $17.1 \mathrm{~m}$. As a result, the original fiber-ring cavity presents a moderately high netnormal dispersion of $0.44 \mathrm{ps}^{2}$. On the other hand, in the Fabry-Perot cavity, see Fig. 1(b) the original configuration had no extra delay line, whereas the PM YDF length was $0.39 \mathrm{~m}$, giving a round-trip cavity length of $4.23 \mathrm{~m}$. Since this cavity is considerably shorter, the net (normal) dispersion of the original Fabry-Perot cavity was considerably lower $\left(0.107 \mathrm{ps}^{2}\right)$. In both cases, these configurations of the cavities included the pigtails of the fiber optics components used (PM WDM, PM OFC, etc.).

Our study begins with a full characterization of the original configurations for both cavities. Next, we experimentally analyzed the effect of a change of the net (normal) dispersion on the output light pulses, taking as a starting point the original configurations. Finally, we also discuss out-of-cavity recompression possibilities.

\section{Results and Discussion}


In the following, we will show the characterization of the original configuration for both cavities. The lasers were self-starting once the pump power reaches the required level, being unnecessary any mechanical perturbation to initiate mode-locking. In each case, we monitored the temporal pulse waveforms, spectra, and intensity autocorrelation traces. The original configuration for the fiber-ring laser consisted of $0.47 \mathrm{~m}$ length of PM YDF and a PM SMF delay line length of $17.1 \mathrm{~m}$, resulting in a total cavity length of $17.57 \mathrm{~m}$; see Fig. 1(a). On the other hand, the Fabry-Perot laser consisted of a $0.39 \mathrm{~m}$ long PM YDF and the rest of PM SMF, resulting in a round-trip cavity length of $4.23 \mathrm{~m}$; see Fig. 1(b).
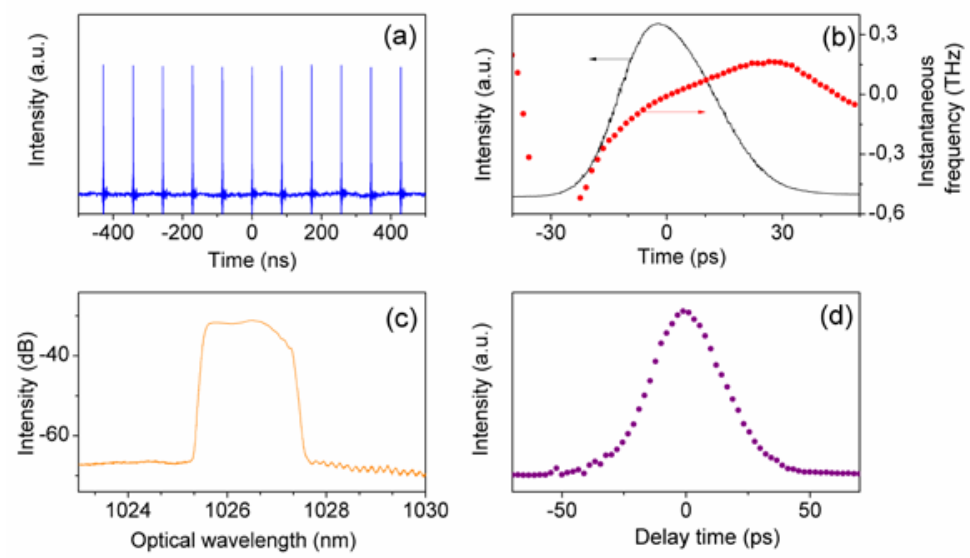

Fig. 2. Fiber-ring laser: (a) temporal trace of the output light train, (b) temporal waveform of one single pulse -left axis- and its corresponding instantaneous frequency profile -right axis- (solid curve and solid scatter points, respectively), (c) output light pulse spectrum, and (d) intensity autocorrelation trace.

Figure 2(a) shows the output light pulses train of the fiber-ring laser, at a frequency of $11.67 \mathrm{MHz}$ for a pump power of $108 \mathrm{~mW}$; as expected, the measured frequency corresponds well with the reciprocal of the round-trip time for the measured cavity length of $17.57 \mathrm{~m}$. In Fig. 2(b) we show the temporal intensity profile of a single light pulse, with an FWHM of 27 ps and measured peak power of $0.784 \mathrm{~W}$ (pulse energy of $21.2 \mathrm{pJ}$ ). On the other hand, the spectrum of the output light pulses is shown in Fig. 2(c). Since the cavity is entirely ANDi, this laser operates at the dissipative soliton regime, as confirmed by the steep spectral edges in the optical spectrum. Without any filtering within the cavity, the laser emits at the central optical wavelength of $1026.51 \mathrm{~nm}$, with an optical bandwidth of $2.1 \mathrm{~nm}$ (due to the steep spectral edges it is more representative to measure at $-10 \mathrm{~dB}$ ). The time-bandwidth product (TBP) in this case is 16 , well above the TBP of $\sim 0.315$ for a transform-limited $\operatorname{sech}^{2}$ pulse, showing that these mode-locked pulses are highly chirped $[22,23]$, which also is a typical signature of the dissipative soliton regime. Therefore, these chirped light pulses could be recompressed up to the limit given by the time-bandwidth product: $\sim 0.5 \mathrm{ps}$. Next, we measured the instantaneous frequency (IF) profile of the output light pulses by using a simple technique previously developed by us $[24,25,26]$. The technique only requires measuring the temporal intensity waveforms at the input and output of a dispersive line -in this case: $160 \mathrm{~m}$ length of PM SMF-, together with the knowledge of the chromatic dispersion of the optical fiber. Therefore, the IF profile is obtained just by using a single equation in a non-iterative single-step numerical calculation, which is shown on the right axis in Fig. 2(b). There, we can observe, in the central section of the light pulse, a linear chirp with a slope of $8.8 \mathrm{GHz} / \mathrm{ps}$. It is also important to distinguish between the DS pulse regime from other regimes like noise-like pulses (NLP), which are irregular bursts with intense fluctuations [27]. NLPs have also a broadband spectrum, but its more distinctive signature is given by the autocorrelation trace, which consists of a broad background with a sharp narrow central peak [28]. With the purpose 
to elucidate this point, we also performed autocorrelation measurements; see Fig. 2(d). There is any trace corresponding with the emission of NLPs; being the duration of the measured pulses -via decorrelation- in correspondence with the value previously measured with the sampling oscilloscope.
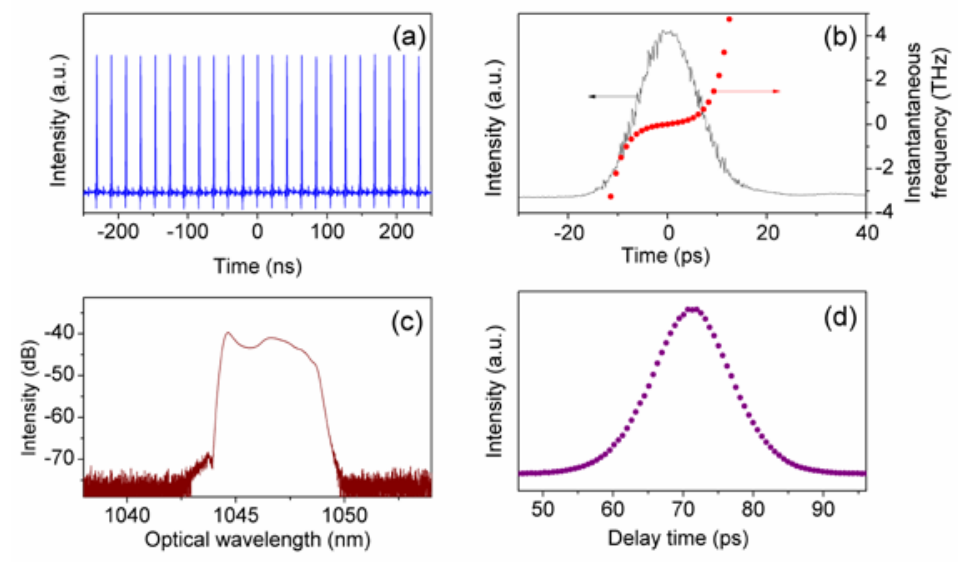

Fig. 3. Fabry-Perot laser: (a) temporal trace of the output light train, (b) temporal waveform of one single pulse -left axis- and its corresponding instantaneous frequency profile -right axis- (solid curve and solid scatter points, respectively), (c) output light pulse spectrum, and (d) intensity autocorrelation trace.

In Fig. 3, we show the characterization of the original configuration of the Fabry-Perot laser. Figure 3(a) shows the output light pulses train at a frequency of $48.54 \mathrm{MHz}$ for a pump power of $101 \mathrm{~mW}$; as expected, the measured frequency corresponds well with the reciprocal of the round-trip time for the measured cavity length of $4.23 \mathrm{~m}$. In Fig. 3(b), we show the sampling oscilloscope trace of one single light pulse, with an FWHM of 13 ps. The instantaneous frequency is also shown in this figure, showing a linear chirp with a slope of 33 $\mathrm{GHz} / \mathrm{ps}$ at the central section of the light pulse. In Fig. 2(d) it is shown the measured temporal intensity autocorrelation trace. After using the decorrelation factor for a sech ${ }^{2}$ profile, it results in pulse duration of $8.3 \mathrm{ps}$; which could be taken as a more precise measurement, since the oscilloscope measurement is near its resolution limit. It should be noted also there is no trace of NLP emission. Finally, the spectrum of the output light pulses is shown in Fig. 3(c); without any filtering within the cavity, the laser emits at the central optical wavelength of $1046.8 \mathrm{~nm}$, with a $-10 \mathrm{~dB}$ bandwidth of $5.9 \mathrm{~nm}$. This laser also operates at the dissipative soliton regime as in the preceding configuration, as confirmed by the steep spectral edges in the optical spectrum. The time-bandwidth product (TBP) is 13, showing that the mode-locked pulses are chirped, which also is a typical signature of the dissipative soliton regime.

As we mentioned in the introduction, different all-PM fiber lasers producing chirped light pulses in the all-normal dispersion regime have been demonstrated using as modelocker: a nonlinear amplifying loop mirror [12, 29], a SESAM [11, 30], a carbon nanotube saturable absorber [18], or a semiconductor SA in transmission [17]. These all-PM fiber lasers produced chirped light pulses with temporal pulse widths of 68 ps [12], 61 ps [29], 7ps [11], 30 ps [30], 1230 ps [18], and 400 ps [17]. As compared with these works, only the work presented in Ref. [18] precludes of an ad hoc frequency limiter, being constructively very similar to our setup shown in Fig. 1(a) -except by the use of a graphene SA as mode-locker, but delivering output light pulses well in the nanoseconds regime. Further, our setups deliver one of the shortest chirped light pulses, well below the tenth-of-ps regime (without de-chirping by out-of-cavity recompression). On the other hand, the measured spectral widths and frequency chirps are comparable also to the preceding works (in the range 1-10 $\mathrm{nm}$ ), retaining 
the potential for out-of-cavity recompression by de-chirping to the sub-picosecond regimes, as we will discuss later.

Next, we analyzed the effect of dispersion on the emission of these lasers; by progressively varying the length of the optical fiber cavity by adding/subtracting PM SMF and/or PM PCF. Therefore, we were able to study the behavior of the fiber-ring laser, when the net-cavity dispersion ranged from the net-anomalous regime $\left(-0.296 \mathrm{ps}^{2}\right)$ up to an ultralong ANDI cavity of $172.5 \mathrm{~m}$ with a net-normal dispersion of $4.33 \mathrm{ps}^{2}$; both values should be compared with the net dispersion of the original fiber-ring cavity $\left(0.44 \mathrm{ps}^{2}\right)$. First, in the netanomalous regime, we did not observe soliton mode-locking; which could be a consequence of the absence of a specific frequency limiter in our setup [8]. Figure 4(a) shows the pulse duration as a function of dispersion. It can be observed how the pulse duration monotonically decreases when the magnitude -i.e., the absolute value- of the chromatic dispersion approaches zero, although with slightly different rates, being slower in the net-normal dispersion regime. From Fig. 4(a), we see that the temporal duration monotonically increases at both sides of the zero of dispersion. The shortest pulses obtained were of 6 ps (peak power of $4 \mathrm{~W}$ ); While the pulses energies ranged from $27 \mathrm{pJ}(7 \mathrm{ps})$ to $115 \mathrm{pJ}$ (162 ps) and $6 \mathrm{pJ}(6$ ps) to $7 \mathrm{pJ}$ (61 ps), in the net-normal and net-anomalous regimes, respectively. In this line, in Ref. [12] the authors studied a PM fiber ring laser in the net-normal regime, and extensive numerical simulations revealed that the output energy and duration can simply be up-scaled through the lengthening of the cavity, in accordance with our results. We also performed a similar experiment for the Fabry-Perot laser, progressively lengthening the optical fiber cavity with PM SMF, but always maintaining a fixed PM YDF length of $0.5 \mathrm{~m}$. Therefore, the value of the net (normal) dispersion in the cavity was increased from the original value of $0.107 \mathrm{ps}^{2}$ up to $5 \mathrm{ps}^{2}$. Figure 4(b) shows the pulse duration as a function of dispersion. It can be observed, that the pulse duration monotonically increases with the chromatic dispersion. However, when the dispersion was raised too much -up to $5 \mathrm{ps}^{2}$ - there is not mode-lock, showcasing the importance of dispersion management for stable dissipative soliton formation. For this reason, Fig. 4(b) presents a considerably narrower excursion in net chromatic dispersion values, as compared with Fig. 4(a). In conclusion, the dependence of the pulse duration as a function of the net cavity dispersion was approximately independent of the selected cavity. Further, the pulse duration was approximately the same at the same values of net dispersion; see Figs. 4(a) and 4(b). Although, from these experiments, the Fabry-Perot cavity would be much more sensitive to large values of dispersion, being not possible a stable mode-locking emission at large net chromatic dispersion values. Perhaps this is surely connected with the higher power losses of the FP cavity of our setup ( $7.4 \mathrm{~dB})$ as compared with the FR laser ( $4 \mathrm{~dB})$. However, there is one key advantage of the Fabry-Perot cavity: it allows the construction of shorter cavities, mainly for three reasons. First, it is not essential to include an optical fiber coupler within the cavity, since the output light pulses can be extracted through the dichroic mirror. Second, the wavelength division multiplexer could be located out of the cavity; by simultaneously using the dichroic mirror as the input port of the laser light pump. And third, it is not necessary to include an isolator within the cavity to ensure unidirectional operation, as in the fiber ring cavity. As a result, this shorter cavity has a lower intracavity net dispersion and thus allowing the formation of a higher frequency train of shorter light pulses. 


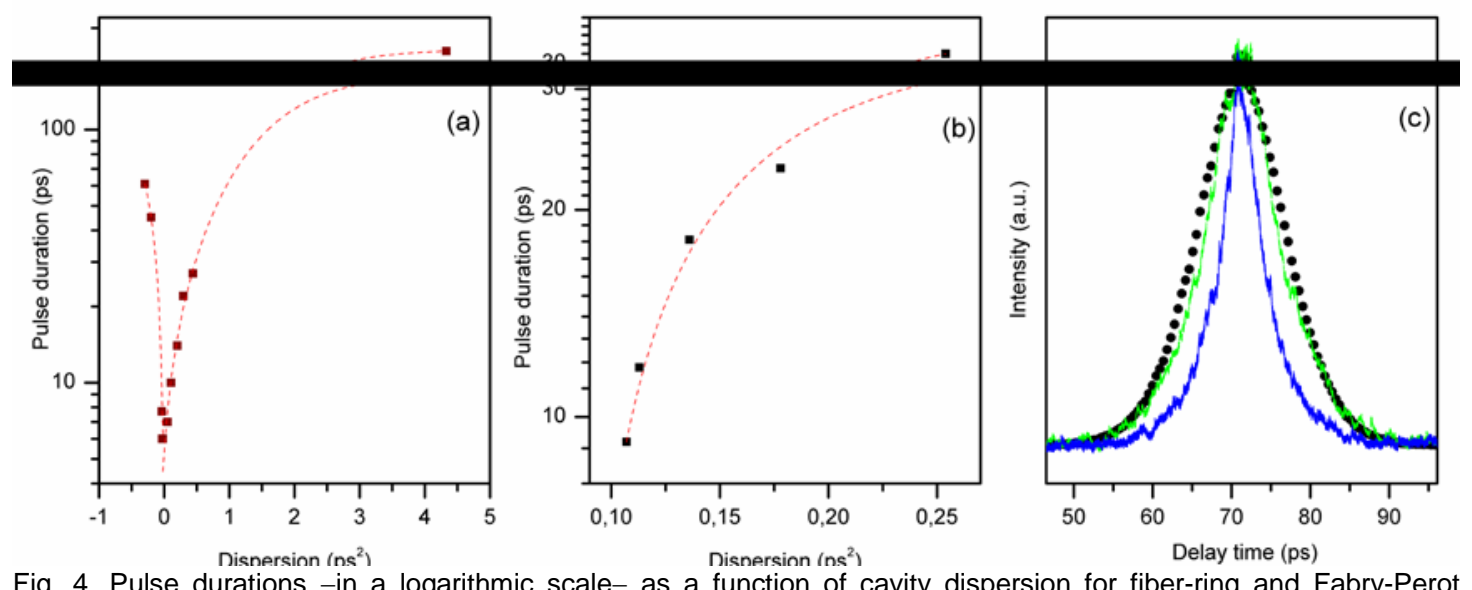

Fig. 4. Pulse durations -in a logarithmic scale- as a function of cavity dispersion for fiber-ring and Fabry-Perot cavities, (a) and (b) respectively; the dashed curves are just a guide to the eyes. (c) Fabry-Perot laser: measured intensity autocorrelation traces at the input of the PM PCF delay line (solid scatter points), and after one single PM PCF pass (green curve), and double PM PCF pass (blue curve).

On the other hand, it is well-known that DSs can be recompressed out of the cavity by further propagation through a dispersive (anomalous) line. As an example, we test this possibility by using the Fabry-Perot laser as a light source. With this purpose, we disposed of a 26 m long PM PCF with either a single, or double-pass transmission possibilities; see Fig. 1(c). The PM PCF has a chromatic dispersion of $17 \mathrm{ps} /(\mathrm{nm} \mathrm{km})$ at $1030 \mathrm{~nm}$; therefore a single or double-pass transmission involves respectively $-0.364 \mathrm{ps}^{2}$, and $-0.728 \mathrm{ps}^{2}$ of chromatic dispersion. Figure 4(c) exemplifies the recompression possibilities for the output light pulses of this laser; to this end, it is showcased simultaneously the intensity autocorrelation traces of the input light pulse, single, and double-pass transmission through the PM PCF line. Therefore an input light pulse with a temporal duration of $8.2 \mathrm{ps}$ is successively narrowed to 5.3 ps, and 3.1 ps after a single and double-pass transmission through the PM PCF delay line. In these experiments, we were constrained by the available length of PM PCF in our laboratory; however, since the TBP of these pulses ranges between 7 and 13 we envisage the limit for recompression of these pulses would be between $190 \mathrm{fs}$ and $360 \mathrm{fs}$.

\section{Conclusions}

We investigated the behavior of a polarization-maintaining passively mode-locked ytterbium-doped laser for two different configurations of the cavity: fiber-ring and Fabry-Perot. It is worth noting that in both setups, the filtering action is solely performed by the spectral overlapping of the different components within the lasers cavity, as opposed to previous works that include some ad hoc frequency limiter. We found that the lack of an ad hoc filter within the cavity does not deteriorate severely the performance, as regarding the production of DSs. We also report the changes in the output light pulses when the net dispersion of the cavity was varied. We found that the Fabry-Perot cavity was lesser permissive to higher net-normal dispersion values, being directly not possible the operation at high net-normal dispersion values, as compared with its fiber-ring counterpart. However, they produce similar light pulses, as regarding temporal width, when both cavities have similar net-normal dispersion values. Additionally, we also performed out-of-cavity recompression by a PM-PCF. This experimentally demonstrates the potential to obtain short light pulses with two simple PM fiber lasers.

\section{Acknowledgements}

This work was supported in part by the European Union, project IPN-Bio (Ref.: H2020-MSCA- 
RISE-2019-872049), and by the Generalitat Valenciana of Spain (Ref.: PROMETEO/2019/ 048). C. Cuadrado-Laborde acknowledges the financial support from project PICT 2015-1828 (FONCYT, Argentina), PIP 11220150100607CO (CONICET, Argentina) and the Programa de Investigadores Invitados de la Universidad de Valencia (Spain).

\section{References}

1 L.F. Mollenauer and R. H. Stolen, "The soliton laser," Opt. Lett. 9, 13-15 (1984).

$2 \mathrm{H}$. Haus and M. Islam, "Theory of the soliton laser," IEEE J. Quantum Electron. 21, 1172-1188 (1985).

3 P. Grelu, and N. Akhmediev, "Dissipative solitons for mode-locked lasers," Nature Photon. 6, 84-92 (2012).

4 A. Chong, J. Buckley, W. Renninger, and F. Wise, "All-normal-dispersion femtosecond fiber laser," Opt. Express 14, 10095-10100 (2006).

5 A. Chong, W.H. Renninger, and F.W. Wise, "All-normal-dispersion femtosecond fiber laser with pulse energy above 20nJ," Opt. Lett. 32, 2408-2410 (2007).

6 M. Brotons-Gisbert et al., "Comprehensive Theoretical and Experimental Study of Short- and LongTerm Stability in a Passively Mode-Locked Solitonic Fiber Laser," J. of Lightwave Technol. 33, 40394049 (2015).

7 X. Shen, W. Li, and H. Zenga, "Polarized dissipative solitons in all-polarization-maintained fiber laser with long-term stable self-started mode-locking," Appl. Phys. Lett. 105, 101109 (2014).

8 C. Cuadrado-Laborde, A. Carrascosa, A. Díez, J.L. Cruz, and M.V. Andrés, "All polarization-maintaining passively mode-locked fiber-ring ytterbium-doped laser; from net-normal to net-anomalous dispersion," Laser Physics 30, 065102 (2020).

9 C. Cuadrado-Laborde, A. Carrascosa, A. Díez, J.L. Cruz, and M.V. Andrés, "All polarization-maintaining passively mode-locked $\mathrm{Yb}$-doped fiber laser: pulse compression using an anomalous polarizationmaintaining photonic crystal fiber," IEEE Photonics Journal 11, 1505409 (2019)

10 C. Aguergaray, R. Hawker, A.F.J. Runge, M. Erkintalo, and N.G.R. Broderick, “120 fs, 4.2 nJ pulses from an all-normal-dispersion, polarization-maintaining, fiber laser," Appl. Phys. Lett. 103, 121111 (2013).

11 J-.B. Lecourt, C. Duterte, F. Narbonneau, D. Kinet, Y. Hernandez, and D. Giannone, "All-normal dispersion, all-fibered PM laser mode-locked by SESAM," Opt. Express 20, 11918-11923 (2012).

12 M. Erkintalo, C. Aguergaray, A. Runge, and N.G.R. Broderick, "Environmentally stable all-PM allfiber giant chirp oscillator," Opt. Express 20, 22669-22674 (2012).

13 B. Ortaç, M. Plötner, J. Limpert, and A. Tünnermann, "Self-starting passively mode-locked chirpedpulse fiber laser," Opt. Express 15, 16794-16799 (2007).

14 C. Aguergaray, N.G.R. Broderick, M. Erkintalo, J.S.Y. Chen, and V. Kruglov, "Mode-locked femtosecond all-normal all-PM Yb-doped fiber laser using a nonlinear amplifying loop mirror," Opt. Express 20, 10545-10551 (2012). 
15 Y. Yu, H. Teng, H. Wang, L. Wang, J. Zhu, S. Fang, G. Chang, J. Wang, and Z. Wei, "Highly-stable mode-locked PM Yb-fiber laser with $10 \mathrm{~nJ}$ in 93-fs at $6 \mathrm{MHz}$ using NALM," Opt. Express 26, 1042810434 (2018)..

16 B. Xu, A. Martinez, S.Y. Set, and S. Yamashita, "All-Polarization Maintaining Fiber Laser and Pulse Compressor," IEEE Photon. Technol. Lett 30, 2151-2154 (2018).

17 X-.S. Xiao, "Low-repetition-rate, all-polarization-maintaining Yb-doped fiber laser mode-locked by a semiconductor saturable absorber," Chinese Phys. B 26, 114204 (2017).

18 R.I. Woodward et al., "Scalar Nanosecond Pulse Generation in a Nanotube Mode-Locked Environmentally Stable Fiber Laser," IEEE Photon. Technol. Letters 26, 1672-1675 (2014).

19 M. Delgado-Pinar, A. Diez, J.L. Cruz and M.V. Andres, "High Extinction-Ratio Polarizing Endlessly Single-Mode Photonic Crystal Fiber," IEEE Photonics Tech. L. 19, 562-564 (2007).

20 M. Delgado-Pinar, A. Díez, S. Torres-Peiró, M.V. Andrés, T. Pinheiro-Ortega, and E. Silvestre, "Waveguiding properties of a photonic crystal fiber with a solid core surrounded by four large air holes," Opt. Express 17, 6931-6938 (2009)

21 R.B. Dyott, Elliptical Fiber Waveguides (London: Artech House, 1995).

22 D.S. Kharenko, O.V. Shtyrina, I.A. Yarutkina, E.V. Podivilov, M.P. Fedoruk, and S.A. Babin, "Highly chirped dissipative solitons as a one-parameter family of stable solutions of the cubic-quintic Ginzburg-Landau equation," J. Opt. Soc. Am. B 28, 2314-2319 (2011).

23 D.S. Kharenko, E.V. Podivilov, A.A. Apolonski, and S.A. Babin, "20 nJ 200 fs all-fiber highly chirped dissipative soliton oscillator," Opt. Lett. 37, 4104-4106 (2012).

24 C. Cuadrado-Laborde, A. Carrascosa, P. Pérez-Millán, A. Díez, J.L. Cruz, and M.V. Andrés. "Phase recovery by using optical fiber dispersion," Opt. Lett. 39, 598-601 (2014).

25 C. Cuadrado-Laborde, M. Brotons-Gisbert, G. Serafino, A. Bogoni, P. Pérez-Millán, and M.V. Andrés. "Phase recovery by using optical fiber dispersion and pulse pre-stretching," Appl. Phys. B 117, 11731181 (2014).

26 C. Cuadrado-Laborde, I. Armas-Rivera, A. Carrascosa, E.A. Kuzin, A. Díez, and M.V. Andrés, "Instantaneous frequency measurement of dissipative soliton resonance light pulses" Opt. Lett. 41 5704-5707 (2016).

27 M. Horowitz, Y. Barad, and Y. Silberberg, "Noiselike pulses with a broadband spectrum generated from an erbium-doped fiber laser," Opt. Lett. 22, 799-801 (1997).

28 C. Aguergaray, A. Runge, M. Erkintalo, and N. G. R. Broderick, "Raman-driven destabilization of mode-locked long cavity fiber lasers: fundamental limitations to energy scalability," Opt. Lett. 38, 2644-2646 (2013)

29 S. Ou, G. Liu, D. Qiu, L. Guo, and Q. Zhang, "All-polarization-maintaining, all-normal-dispersion mode-locked fiber laser with spectral filtering in a nonlinear optical loop mirror," Opt. Express 28, 23670-23678 (2020). 
30 M. Baumgartl, J. Abreu-Afonso, A. Díez, M. Rothhardt, J. Limpert, and A. Tünnermann, "Environmentally stable picosecond Yb fiber laser with low repetition rate," Appl. Phys. B 111, 39-43, (2012). 